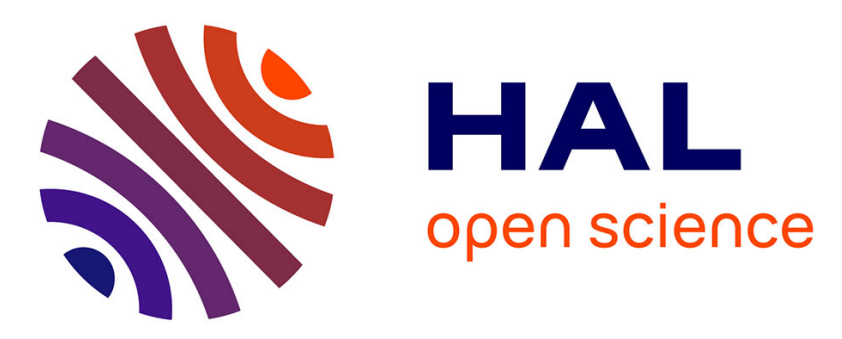

\title{
Intra-crystalline mesoporous SAPO-11 prepared by a grinding synthesis method as FCC promoters to increase iso-paraffin of gasoline
}

\author{
Yuxiang Liu, Wanrong Liu, Yuchao Lyu, Xinmei Liu, Jun Zhang, Yu Gu, \\ Jiankun Huang, Svetlana Mintova, Zifeng Yan
}

\section{To cite this version:}

Yuxiang Liu, Wanrong Liu, Yuchao Lyu, Xinmei Liu, Jun Zhang, et al.. Intra-crystalline mesoporous SAPO-11 prepared by a grinding synthesis method as FCC promoters to increase iso-paraffin of gasoline. Microporous and Mesoporous Materials, 2020, 305, pp.110320. 10.1016/j.micromeso.2020.110320 . hal-02893786

\section{HAL Id: hal-02893786 https://hal.science/hal-02893786}

Submitted on 27 Nov 2020

HAL is a multi-disciplinary open access archive for the deposit and dissemination of scientific research documents, whether they are published or not. The documents may come from teaching and research institutions in France or abroad, or from public or private research centers.
L'archive ouverte pluridisciplinaire HAL, est destinée au dépôt et à la diffusion de documents scientifiques de niveau recherche, publiés ou non, émanant des établissements d'enseignement et de recherche français ou étrangers, des laboratoires publics ou privés. 


\title{
Intra-crystalline MesoporousSAPO-11 Prepared by a Grinding
}

\section{SynthesisMethodas FCC Promoters to Increase iso-Paraffin of Gasoline}

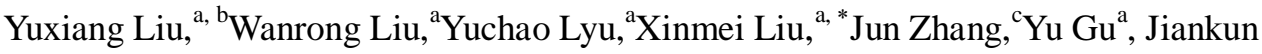

\author{
Huang, ${ }^{\text {a }}$ Svetlana Mintova, ${ }^{\mathrm{a}}$, Zifeng Yan $^{\mathrm{a}}$
}

${ }^{a}$ State Key Laboratory for Heavy Oil Processing, China University of Petroleum, Qingdao, 266580,

China

${ }^{\mathrm{b}}$ College of Chemical Engineering, Qingdao University of Science \& Technology, Qingdao 266042, China

${ }^{\mathrm{c}}$ College of Material Science and Engineering, China University of Petroleum, Qingdao, 266580 P. R. China

${ }^{\mathrm{d}}$ Laboratoire Catalyse \& Spectrochimie, ENSICAEN, Université de Caen, 14000, Caen, France 


\begin{abstract}
Ahierarchical SAPO-11 was prepared by a grinding synthesis method (GSM) and added as a promoter to increase the iso-paraffin yield of gasoline in fluid catalytic cracking (FCC) reaction. Compared tothe classical hydrothermal method, the GSM leads to thecreation of intra-crystalline mesopores with the assistance of a surfactant (Cetyltrimethyl Ammonium Bromide, CTAB). CTAB acts asa structure directing agentand as a hard template during the crystallization of SAPO-11. CTAB micelles formedthe intra-crystalline mesoporesin the SAPO-11 crystals. Theintra-crystalline mesoporosity of the SAPO-11is preservedat $800{ }^{\circ} \mathrm{C}$ understeaming, which render the materialas an appropriate component of the FCC catalyst.Besides, the presence of mesopores provides isomerization active sites and accelerates the desorption of iso-paraffin. The mesoporous SAPO-11 showed a higher selectivity for iso-paraffin and the yield of iso-paraffin increased by $2 \%$.
\end{abstract}




\section{Introduction}

The fluid catalytic cracking(FCC) reactionis a complexparallel-consecutive reaction system consisting cracking, isomerization, aromatization, and hydrogen transfer reactions[1].In FCC, feedstock (e.g., vacuum gas oil) iscracked to primary cracking intermediates. The primary cracking intermediates are re-cracked to smaller molecules (e.g., gasoline and liquefied petroleum gas). As for gasoline fractions, isomerization, aromatization, and hydrogen transfer reaction occurto affect the components of gasoline (i.e., n-/iso-paraffin, n-/iso-olefin, naphthenic hydrocarbon, and arene).ZSM-5 promoter increasethe octane number of gasoline by generating olefin and arene [2]. However, the content of olefin and arene are restricted for environmental considerations (e.g., controlling emission of $\mathrm{NO}_{\mathrm{X}}$ ). Therefore, iso-paraffin is an ideal component for gasoline with a high octane number.

Compared toZSM-5, when SAPO-11 is added as promoter, it hasa high isomerization activity due to mild acidities and one-dimensional 10-membered-ring channels(i.e., $0.39 \mathrm{~nm} \times 0.63 \mathrm{~nm})$ [3].Isomerization reaction is following the"pore-mouth" reaction mechanism [4]. Catalytic conversion and selectivity for isomers are closely related to the pore mouth of micropores, therefore, it is important to increase the contactof microporeswith reagents.Decreasing particle size of catalysts lead to an increase of the external surface area as well as introducing hierarchical porescan alter the exposure of micropores. For example, the yield of iso-paraffin was increased by $\sim 5.0 \mathrm{wt} \%$ when the particle size of SAPO-11 catalysts decreased from 2000 to 400 $\mathrm{nm}[3]$. While introducing hierarchical pores in thestructure has the following main 
advantages: (1) decrease ofdiffusion resistance and increase of the utilization of micropore, and (2) extending the lifetime of catalysts especially for those thatdeactivate due to coke formation in micropores (e.g., deactivation of SAPO-34 catalysts in the methanol to olefins reaction)[5]. When the mesopores are embedded into a catalyst(i.e., intra-crystalline mesopores), the catalystpossesses a higher hydrothermal stability.

One of the commonly usedapproachesto create intra-crystalline mesopores is the 'bottom-up' method using hard and/or soft templates as mesopore fillers[6]. This approach preserves the crystallinity of the materials[7] and allows tailoring of the pore architecture of molecular sieves[8]. However, the 'bottom-up' approach used for the preparation of mesoporous zeolites has the following particularities: a phase separation between the hydrophobic template and the hydrophiliczeolitegeloccurs due to the weak interactions between them, which is commonly observed in both hard and soft mesopore templated systems [9]. Efforts in using steam-assisted-conversion method[10], synthesis at adaptable $\mathrm{pH}$ of precursor gel[11], and costlydesign of specific mesopore templates[12] are used to address the above issues. Exquisiteandtime-consuming operations[13, 14] are needed but limit the commercial implementation of the methods applied toward the preparation of the mesoporous zeolites.

In the present work,the high yield intra-crystallineSAPO-11 materials were prepared viaa grinding synthesis method (GSM) with the assistance of cetyltrimethyl ammonium bromide $(\mathrm{CTAB})$ as a mesoporous filler [15]. It is worth noting that the 
GSM does not need extra solvent for mixing the precursors and transferring amorphous substance to crystalline materials. It also decreases the amountof amine-containing wastewater.Witha shorterlength of carbon chain, n-dodecane has a relative lower reaction activity compared to components with a long carbon chain length and was selected as a model compound of primary cracking intermediatesto investigate the effect of SAPO-11 promoteron the reactions (e.g., cracking and isomerization) under FCC conditions.

\section{Experimental}

2.1 Synthesis of intra-crystallineSAPO-11 viaa grinding synthesis method (sample GSM-CTAB@0.1)

Pseudo-boehmite (1.45 g, Industrial grade, 70 wt.\% $\mathrm{Al}_{2} \mathrm{O}_{3}$, Yantai Henghui Petrochemical Co., Ltd), phosphoric acid (2.31 g, Analytical reagent, 85 wt.\% $\mathrm{H}_{3} \mathrm{PO}_{4}$, Xirong Petrochemical Co., Ltd), di-propylamine (DPA) (1.11 g, Chemical reagent, Sinopharm Chemical Reagent Corporation), colloidal silica (0.80 g, Industrial grade, 30 wt.\% $\mathrm{SiO}_{2}$, Qingdao Haiyang Petrochemical Co., Ltd), and Cetyltrimethyl Ammonium Bromide (CTAB) (0.10 g, Chemical reagent, Sinopharm Chemical Reagent Corporation) were mixed in a mortar. After grinding for $5 \mathrm{~min}$, the amorphous mixture was placed in an autoclave and subjected to crystallization (200 ${ }^{\circ} \mathrm{C}, 24 \mathrm{~h}$ ). The final product was washed by deionized water and activated at $600{ }^{\circ} \mathrm{C}$ for $4 \mathrm{~h}$.

2.2 Synthesis of SAPO-11 viaa grinding synthesis method (sample GSM-CTAB@0) 
Protocol of GSM-CTAB@0 was described as similar with that of GSM-CTAB@0.1. The only difference is without adding CTAB.

2.3 Synthesis ofSAPO-11 viaconventional hydrothermal method (sample HT-CTAB@0.1)

The chemical composition of the amorphous mixture applied is similar with GSM-CTAB@0.1. Pseudo-boehmite (4.37 g) was dispersed in deionized water with stirring for $10 \mathrm{~min}$. Then phosphoric acid (6.92 g), DPA (4.39 g), colloidal silica (2.40 $\mathrm{g})$, and $\mathrm{CTAB}(0.30 \mathrm{~g})$ were added to the solution. Before adding any raw materials, a stirring for 2 hours is needed to make the amorphous mixture homogeneously. After regulating $\mathrm{pH}$ value to 5.5 by adding the DPA or ammonium hydroxide (Chemical reagent, Sinopharm Chemical Reagent Corporation), the amorphous mixture was placed in an autoclave and subjected to crystallization $\left(200{ }^{\circ} \mathrm{C}, 24 \mathrm{~h}\right)$. The final product was washed by deionized water and activated at $600{ }^{\circ} \mathrm{C}$ for $4 \mathrm{~h}$.

\subsection{Hydrothermal treatment of samples GSM-CTAB@0.1 and HT-CTAB@0.1}

Samples were treatedat $800^{\circ} \mathrm{C}$ for $4 \mathrm{~h}$ with a flow of deionized water at the rate of $1.0 \mathrm{~g} \mathrm{~min}^{-1}$ at atmospheric pressure. Sampleswere put in ceramic bowls at $\mathrm{N}_{2}$ atmosphere. When the temperature reached $800^{\circ} \mathrm{C}, \mathrm{N}_{2}$ is switched to a steam $(100 \%)$.

\subsection{Characterizations.}

XRD patterns were measured with an $X^{\prime}$ Pert PRO MPD diffractometer ( $\mathrm{P} A$ Nalytical B.V. Netherlands) with $\mathrm{Cu} \mathrm{K \alpha}$ radiation $(\lambda=0.15418 \mathrm{~nm})$, operating at 40 $\mathrm{kV}, 40 \mathrm{~mA}$, and scanning speed of $10^{\circ} \mathrm{min}^{-1}$. Relative crystallinity is calculated by the sum of peaks areas at $2 \theta=8 \cdot 0-8 \cdot 5^{\circ}$ and $20 \cdot 0-23.5^{\circ}$. The morphology of the samples 
was observed by a scanning electron microscope (Hitachi S-4800, Japan HQ) with an acceleration voltage of $0.5-30 \mathrm{kV}$. The samples were covered by a conductive layer (gold). At least 30 particles were randomly selected to calculate the mean particle diameter (D) by measuring at three different directions. The average of the three values was used to represent the final particle size.

$$
\begin{aligned}
& \mathrm{D}=\mathrm{d} \pm \mathrm{SE} \\
& \mathrm{SE}=\sqrt{\frac{\sum_{1}^{\mathrm{N}}\left(\mathrm{x}_{\mathrm{i}}-\mathrm{d}\right)^{2}}{\mathrm{~N}-1}}
\end{aligned}
$$

d: mean value of selected particles;

SE: standard error;

$\mathrm{N}$ : number of particles;

$\mathrm{x}_{\mathrm{i}}$ : the particle size of specific particles $(\mathrm{i} \leq \mathrm{N})$.

$\mathrm{N}_{2}$ adsorption-desorption measurements were carried out with an ASAP 2020 Micromeritics instrument. Brunauer-Emmet-Teller (BET) surface area and total pore volume were calculated based on the amount of the nitrogen adsorbed at $P / P_{0}=$ 0.05-0.2 and $P / P_{0}=0.99$, respectively. Micropore volume was obtained by the t-plot analysis. Mesopore size distribution was calculated by BJH method. ${ }^{29}$ Si magic angle spinning nuclear magnetic resonance (MAS NMR) spectra were measured with a Bruker Advance 400 spectrometer. Gaussian peak deconvolution was employed to analyze the silicon species. Transmission electron microscopy (TEM) images of samples were obtained with JEOL JEM2100UHR instrument. The samples (thickness, 90-120 nm) were prepared by a Reichert-Jung ultramicrotome (Leica Mikrosysteme Bensheim, Germany).X-ray photoelectron spectroscopy (XPS) was measured with a 
PHI 500 spectrometer using Al Ka radiation. All binding energies were determined with respect to the $\mathrm{C} 1 \mathrm{~s}$ line $(284.8 \mathrm{eV})$ originating from adventitious carbon. High angle annular dark field scanning transmission electron microscopy (HAADF-STEM) imaging and element mapping were carried out using a FEI Titan G2 80-200 TEM/STEM with ChemiSTEM Technology operating at 200kV. The elemental mapping was obtained by energy dispersive $\mathrm{X}$-ray spectroscopy using the Super-X detector on the Titan with a probe diameter of $1 \mathrm{~nm}$ and probe current of $0.4 \mathrm{nA}$. Sampling spot size, depth and uncertainty are $7,110 \mathrm{~mm}$, and $20 \mu \mathrm{s}$, respectively. Temperature-programmed desorption of ammonia ( $\left.\mathrm{NH}_{3}-\mathrm{TPD}\right)$ was conducted to determine the acidity of samples with a ASAP 2920, Micromeritics chemisorption instrument.Brønsted (B) and Lewis (L) acid sites were distinguished by infrared spectroscopy of chemisorbed pyridine (Py) molecules detected on a Nicolet 6700 spectrometer. Samples were dried at $300{ }^{\circ} \mathrm{C}$ for 4 hours and pyridine adsorption was operated at room temperature for $24 \mathrm{~h}$. Desorption of Py was set at $150{ }^{\circ} \mathrm{C}$ to remove the physical adsorbed Py molecules.Template removal and coke deposition on catalysts were analyzed on a Mettler Toledo TGA-2 SF thermal differential analyzerat a heating rate of $10{ }^{\circ} \mathrm{C} / \mathrm{min}$.

\subsection{Catalytic test}

FCC equilibrium catalysts (80-180 mesh) were obtained from Huicheng petrochemical technology co. LTD. As-synthesized SAPO-11 molecular sieves were sieved to 80-180 mesh and added in FCC equilibrium catalysts as a promoter. The amount of promoter added to the commercial FCC catalysts was $10 \mathrm{wt} \%$.It is worth 
noting that the effect of space time with and without SAPO-11 promoter on the investigation of promoter effect has been eliminated in our previous research [16].Catalytic experiments were carried out in a micro-activity test (MAT) fixed bed. The reactions were carried out at $500 \pm 5{ }^{\circ} \mathrm{C}$ and the catalyst to oil ratio was $3 \mathrm{~g} / \mathrm{g}$. Gaseous product (i.e., $\mathrm{C}_{1-5}$ ) was collected by a gas bag. Liquid product (i.e., $>\mathrm{C}_{5}$ ) was cooling in an ice bath. Mass balance for each reaction was calculated (Eq. 3) and only mass balances $>95 \%$ (i.e., the collectmass of $\mathrm{C}_{1-11}$ and deposited coke on catalysts) were considered.

In order to test the isomerization activity of the SAPO-11 promoter, gasoline ranged n-dodecane (Aladdin, $98 \mathrm{wt} \%$ ) was selected as a model compound.Feed was injected within $75 \mathrm{~s}$ andgaseous products were collected for chromatography analysis with one FID detector and two TCD detectors (Varian Inc./ CP3800). Liquid products were measured by composition analysis (i.e., paraffins, olefins, cycloparaffin, and aromatics) using a Varian CP-3800 GC equipped with a CP-Sil PONA CB silica column with a FID detector. Coke content of spent catalyst was quantified using an elemental analyzer (Antek 9000). Conversion and yield were calculated based on Eqs. 4 and 5, respectively.The maximum errors ofeachcomponentare defined as the maximum deviation from the mean valuebased on three parallel experiments.

$$
\begin{gathered}
\text { Mass balance }=\frac{\text { Mass of gases }+ \text { Mass of liquids }+ \text { Mass of coke }}{\text { Mass of feed } \mathrm{C}_{12}} \times 100 \% \text { (Eq. 3) } \\
\text { Conversion }=\frac{\text { Mass of feed } \mathrm{C}_{12}-\text { Mass of } \mathrm{C}_{12} \text { in liquid product }}{\text { Mass of feed } \mathrm{C}_{12}} \times 100 \% \text { (Eq. 4) } \\
\text { Yield }= \\
=\frac{\text { Mass of pruduct }}{\text { Mass of feed } \mathrm{C}_{12}} \times 100 \%
\end{gathered}
$$

\section{Results and Discussions}




\subsection{Phase structure}

XRD patterns of samples synthesized in the presence of CTAB, HT-CTAB@0.1 and GSM-CTAB@0.1, are shown in Figure 1. All Bragg characteristic peaks are attributed to the AEL type structure.The high purity of both samples HT-CTAB@0.1 and GSM-CTAB@0.1 is demonstrated, howeverthe relative peak intensity of some crystal planes is different. For example, the (110) crystal plane in GSM-CTAB@0.1 is weaker in comparison to the peaks at8.5degree. It suggests that oriented crystal growth occurs due to the variation of $\mathrm{pH}$ of the precursor mixture and the solid to liquid ratio.
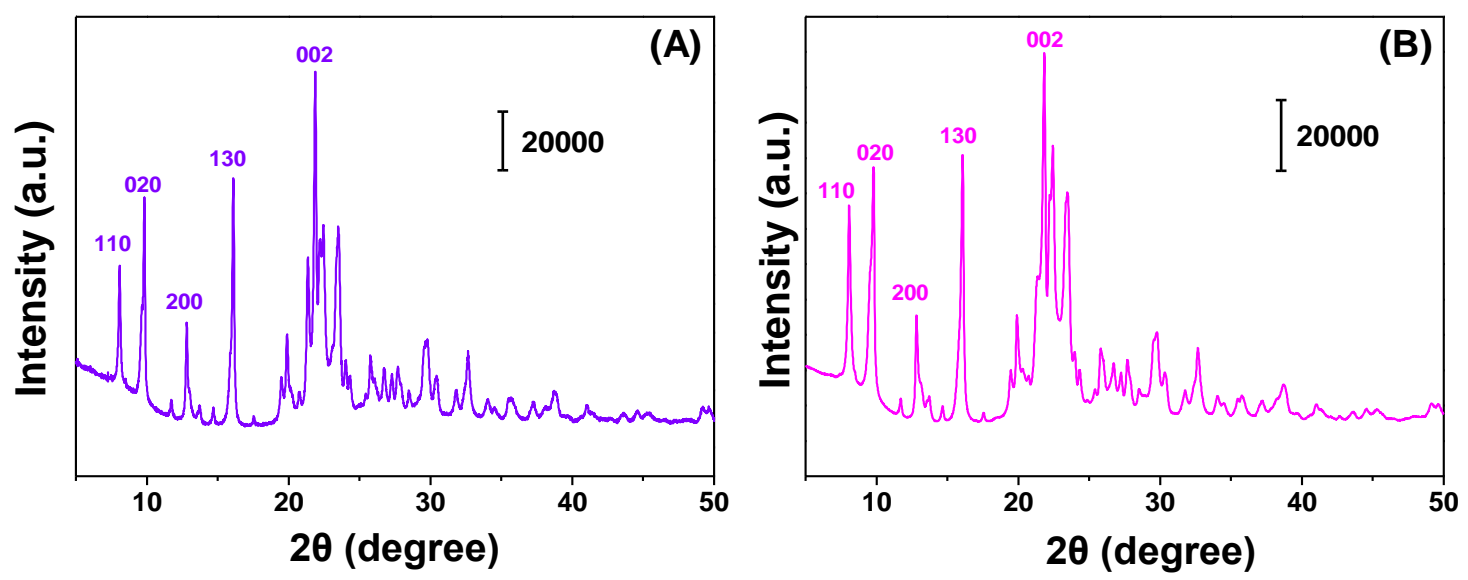

Figure 1. XRD patterns of GSM-CTAB@0.1 (A) and HT-CTAB@0.1 (B) samples

\subsection{Morphology}

Morphology of samples is studied by SEM (Figure 2). Both samples contain irregular particles with spherical morphology. Particle size decreases from $10 \mu \mathrm{mfor}$ HT-CTAB@0.1 (Figure 2A) to $5 \mu \mathrm{m}$ forGSM-CTAB@0.1 (Figure. 2C).The HT-CTAB@0.1 crystals have well shaped facets(Figure 2D), while 
GSM-CTAB@0.1 crystals are smallerwith less pronounced facets (Figure 2B).

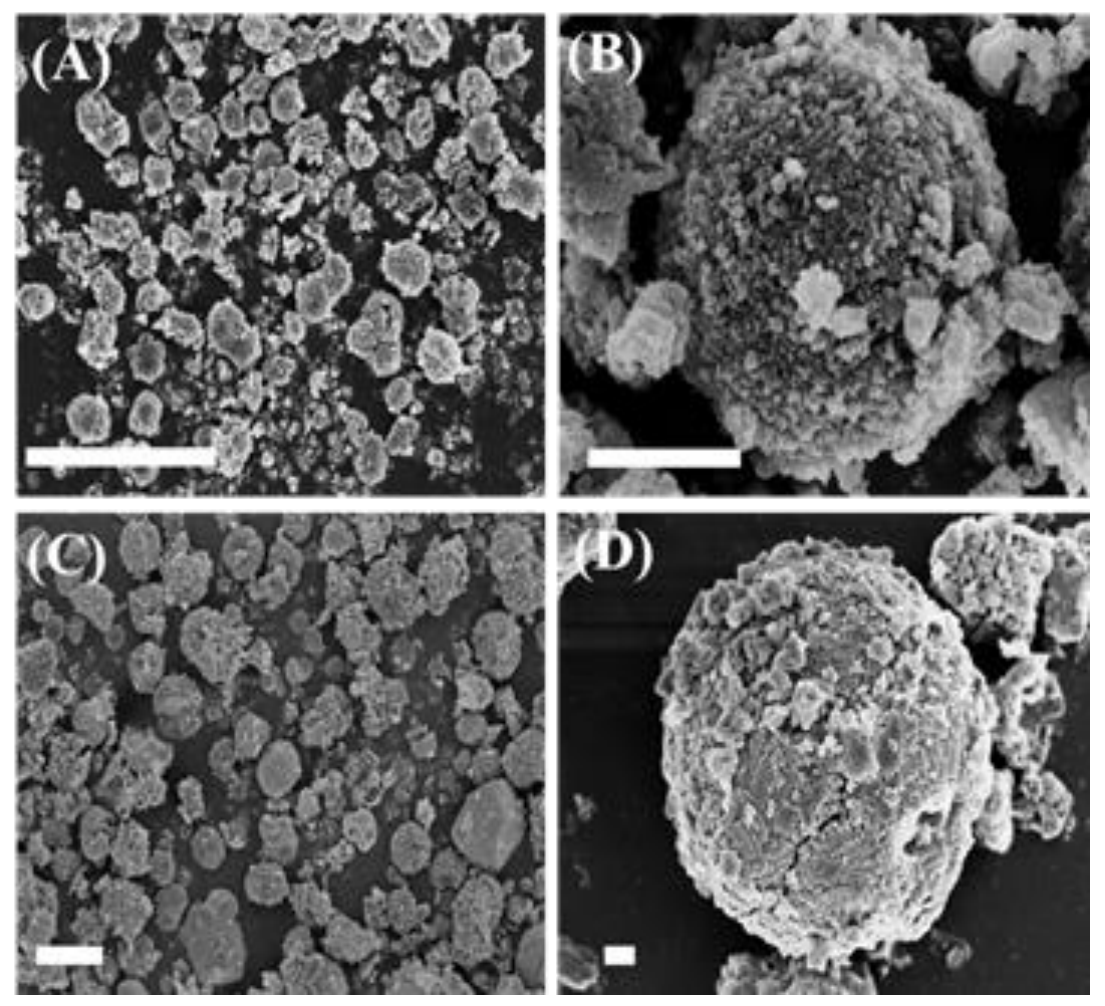

Figure 2. SEM images of GSM-CTAB@0.1 (A and B) and HT-CTAB@0.1 (C and D) at low (A and C) and high (B and D) magnifications: scale bars $\mathrm{M}=10 \mu \mathrm{m}$ in images $\mathrm{A}$ and $\mathrm{C}$ and $\mathrm{M}=2 \mu \mathrm{m}$ in images $\mathrm{B}$ and $\mathrm{D}$, respectively.

\subsection{Dispersion and coordination environment of silicon atoms}

Silicon atoms can isomorphous substitute phosphorus or aluminium atoms, which affect the acidity [17], stability [18], and catalytic performance [19] of SAPO molecular sieves. The dispersion of silicon atoms in the samples is investigated by the STEM and EDS (Figure 3). The Si is homogeneously distributed in the crystalline particles.The elemental composition of the GSM-CTAB@0.1 and HT-CTAB@0.1samples is similar (Table S1). However, the distributionof silicon atoms is different for samplesGSM-CTAB@0.1 and HT-CTAB@0.1. TheEDS results from the external surface and the inner part (bulk)of samples HT-CTAB@0.1 and GSM-CTAB@0.1 are shownin Figure 3A,B.A similar Si/Al ratio on the surface and in 
the bulk of the HT-CTAB@0.1sample is measured. As for GSM-CTAB@0.1, the $\mathrm{Si} / \mathrm{Al}$ ratio on the surface is larger than that in the bulk. This indicates that due to a low concentration of water in the precursor mixture, a non-uniform dispersion of silicon in the sample GSM-CTAB@0.1 is attained.

${ }^{29} \mathrm{Si}$ MAS NMR spectra were collected for the samples in order to investigate the chemical environment of silicon (Figure 4). Peaks I-V at -90 , $-100,-107,-115$, and $-120 \mathrm{ppm})$ are attributed to the coordination of $\mathrm{Si}(4 \mathrm{Al})$, Si (3Al, 1Si), Si (2Al, 2Si), Si (1Al, 3Si), and $\mathrm{Si}$ (4Si), respectively[24]. As shown in Table S2, the concentration of Si (4Al) in the GSM-CTAB@0.1 is $12.3 \%$ less than that in the HT-CTAB@0.1. Compared to sample HT-CTAB@0.1, a 7.8\% higher concentration of $\mathrm{Si}(4 \mathrm{Si})$ in the GSM-CTAB@0.1 is measured possibly associated with the presence of silicon island. This confirms the non-homogeneous distribution of silicon in the sampleGSM-CTAB@0.1 using GSM. This isconsistent with the enrichment of silicon on the surface of the sample (see Figure 3). In the sample prepared under hydrothermal synthesis conditions (HT-CTAB@0.1), the silicon mostly present as monomeric species are highly diluted in the aqueous precursor mixture. These silicon monomeric speciesare preferablyincorporated into zeolitic framework following the SM II rule (i.e., one silicon atom replaces one phosphorus atom) [3].While in the GSM-CTAB@0.1 sample, the higher concentration of silicon atoms resulted in the isomorphous substitution of both aluminum and phosphorus atoms by two silicon atoms (i.e., SM III rule) [3]. 
The silicon distribution is not homogeneous (e.g., concentration of silicon island is $47.6 \%$ ) in the sample previously prepared by the GSM approach [15]. TheCTAB decreasesthe concentration of silicon island from 47.6 to $18.0 \%$. (Table S2). In summary, the CTAB has two functions in the GSM synthesis: (1) it creates mesopores; and (2) ithas structure-directing function [17] that leads tomore homogenous silicon distribution in the samples.

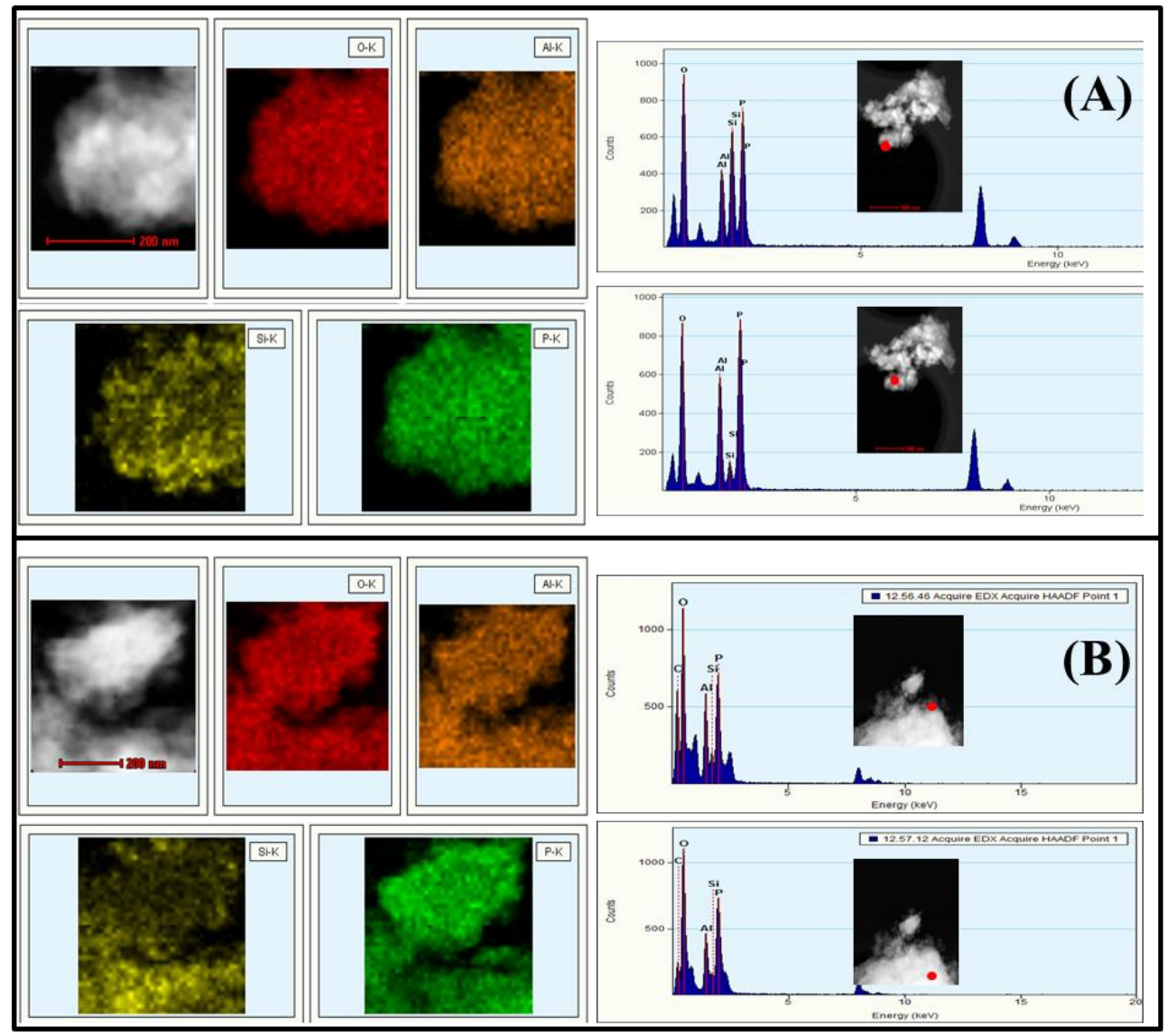

Figure 3. STEM and EDS elemental mapping images of GSM-CTAB@0.1 (A) and HT-CTAB@0.1 (B). Red points in EDS images represent the analysed zones. 

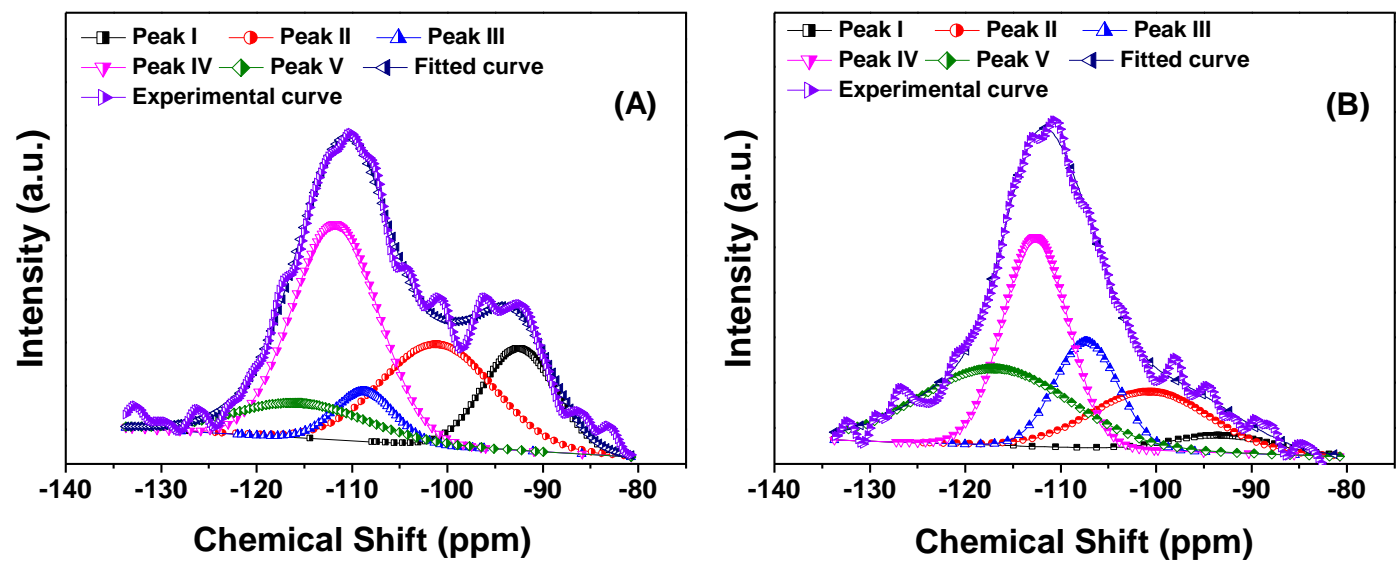

Figure 4. ${ }^{29}$ Si MAS NMR spectra of samples GSM-CTAB@0.1 (A) and HT-CTAB@0.1 (B). (Peak I at -90 ppm, Peak II at -100 ppm, Peak III at -107 ppm, Peak IV at -115 ppm, and Peak V at -120 ppm)

\subsection{Pore structure}

Both samples(GSM-CTAB@0.1 and HT-CTAB@0.1)exhibit a mix Type I and IV isotherms indicating their hierarchical pore structure. A hysteresis loop at a relativepressure of 0.48 is measured which is suggestingthe hierarchical pore structure for both samples (Figure 5).As shown in Table S3, the BET surface areas of samples GSM-CTAB@0.1 and HT-CTAB@0.1 are comparable. The total pore volume of HT-CTAB@0.1 $\left(0.27 \mathrm{~cm}^{3} / \mathrm{g}\right)$ is larger than that ofGSM-CTAB@0.1 $\left(0.21 \mathrm{~cm}^{3} / \mathrm{g}\right)$. The micropore volume is almost identical for both samples HT-CTAB @0.1 $\left(0.06 \mathrm{~cm}^{3} / \mathrm{g}\right)$ and GSM-CTAB@0.1 $\left(0.07 \mathrm{~cm}^{3} / \mathrm{g}\right)$. The BJH pore size distribution curves of GSM-CTAB@0.1 and HT-CTAB@0.1 are shown in inset a' and b' (Figure 5), respectively. Two types of mesopores centered at $\sim 4.1$ and $\sim 20 \mathrm{~nm}$ in GSM-CTAB@0.1 are measured, while a wide range of mesoporesand low pore volume forHT-CTAB@0.1 are measured. Based on the $\mathrm{N}_{2}$ sorption and SEM results, we can conclude that the GSM method leads to the creation of mesopores and also decreasesthe crystalssize. 


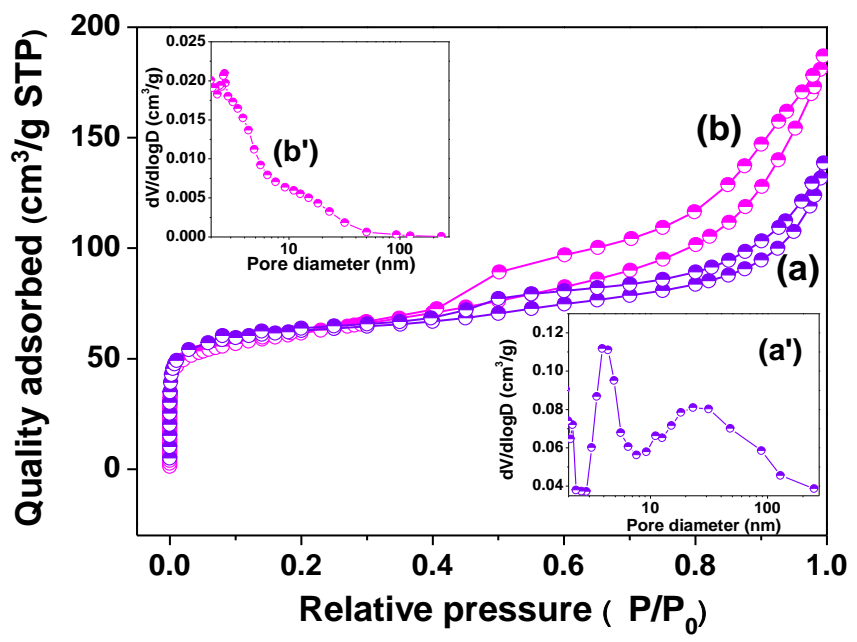

Figure 5. $\mathrm{N}_{2}$ sorption isotherms of GSM-CTAB@0.1 (a) and HT-CTAB@0.1 (b). (Inset: a' and b' are BJH pore size distribution curves based on the adsorption isotherm)

Crystalline structure and porosity of the samples were further investigated by TEM. Compared to the slit pores in sample HT-CTAB@0.1 (Figure S1), two types of mesopore in the GSM-CTAB@0.1 areobserved. The spherical particles were cut into ellipse or round sections prior the TEM characterization (Figure S2A). A section with a long axis length $2.7 \mu \mathrm{m}$ is shown in Figure 6. Based on the $\mathrm{N}_{2}$ sorption results, two kinds of mesopores exist. One is banding mesopore (coloured by blue in the purple region) and the other one is sphere type mesopore (coloured white in the green region). The former is described as inter-crystalline mesopore, which is formed as slit pores among irregular crystals. The sphere intra-crystalline mesopore is attributed to the removal of CTAB micelles and these mesopores are well dispersed parallel to the cutting direction. As shown in the coloured region in Figure 6C, some sphere mesoporesare connected. Therefore, a wide pore size distribution was measured (Figure 6).In addition, the mesopores are uniformly distributed in the GSM-CTAB@0.1at different penetration depths perpendicular of the cutting 
direction (Figure S2 B,C). It indicates that the CTAB micelles are highly dispersed in the amorphous mixture used for the synthesis of SAPO-11 molecular sieves.

TG analyses were carried out on in order to follow the CTAB removalfrom samplesusing GSM and HT methods (Figure 7).Stages I, II, and III represent the remove of adsorbed water, a great mass of CTAB, and DPA, respectively. As for stage IIof GSM-CTAB@0.1, the descending slope is similar with that of HT-CTAB@0.1 ( 150- $250{ }^{\circ} \mathrm{C}$ marked by a purple shadow area). However, the descending slope becomes smaller in the GSM-CTAB@0.1 ( 250-350 ${ }^{\circ} \mathrm{C}$ marked by a grey shadow area). Thisindicates that higher temperature is needed to remove the CTAB micelles fromthe GSM-CTAB@0.1 sample. While the $\mathrm{CTAB}$ are decomposed at lower temperature from sample HT-CTAB@0.1. These results are consistent with the failure of creating intra-crystalline mesopores. 

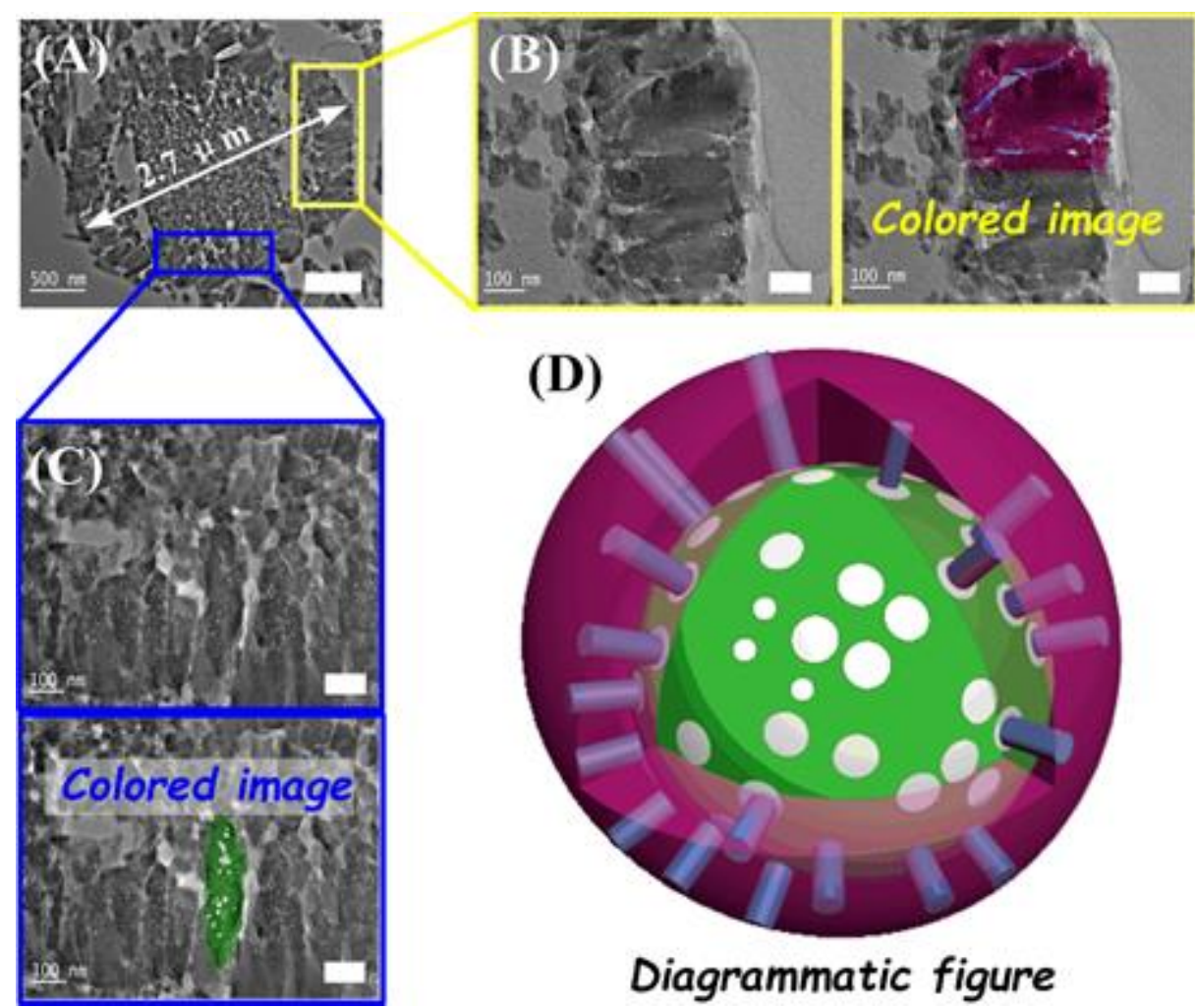

Diagrammatic figure

Figure 6.TEM images of sample GSM-CTAB@0.1 (A, M=500 nm), (B, M=100 nm) and $(C, M=100 \mathrm{~nm})$. Schematic presentation of the pore structure of GSM-CTAB@0.1 deduced by the TEM (D). Two types of mesopore are present in the GSM-CTAB@0.1 crystals shown in blue and white. Note that purple shell and green core represent the same materials. Colours are used to highlight the two types of mesopore only.

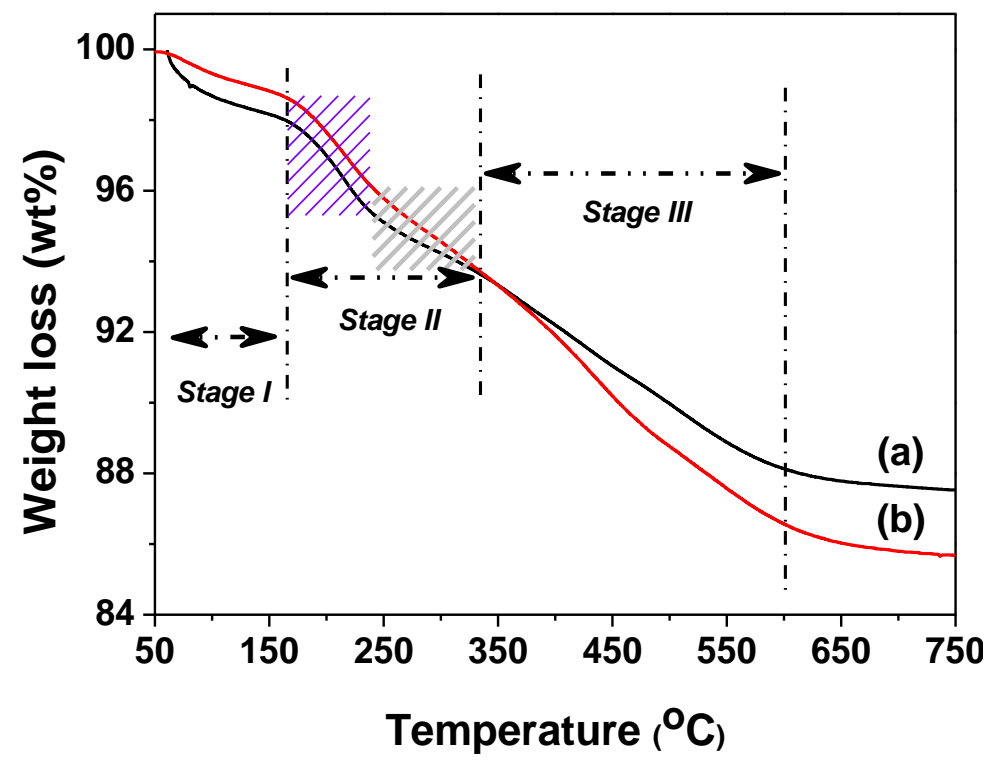

Figure 7. TG curves of samples GSM-CTAB@0.1 (a) and HT-CTAB@0.1 (b).

3.5 Hydrothermal stability of porosity 
The unique bimodal mesopore structure is obtained in GSM-CTAB@0.1.The hydrothermal stability of samples GSM-CTAB@0.1 and HT-CTAB@0.1 after exposure to $100 \%$ steam at $800{ }^{\circ} \mathrm{C}$ for $4 \mathrm{~h}$ was studied;the crystallinity of both GSM-CTAB@0.1 and HT-CTAB@0.1 samplesremains the same. This indicates that introduction of intra-mesopores in the GSM-CTAB@0.1 has a negligible effect on the hydrothermal stability of the structure. Besides the crystalline stability, the effect of hydrothermal treatment on the porosity of sample GSM-CTAB@0.1 and HT-CTAB@0.1 is shown in Figure S3. The decrease of $\mathrm{N}_{2}$ adsorption in micropores of $P / P_{0}<0.05$ is observed due to the destruction of micropores $[17,20]$.The $\mathrm{BJH}$ curves for samples HT-CTAB@0.1 and GSM-CTAB@0.1 after the testare shown in Figure 8A.Inter-crystalline mesopores in HT-CTAB@0.1 and GSM-CTAB@0.1 disappear due to a poor hydrothermal stability.In contrast, intra-crystalline mesopores $(\sim 20 \mathrm{~nm})$ in the GSM-CTAB@0.1 remain.TEM images show a well preserved intra-crystalline mesopores after the stability test (Figure S4).The intra-crystalline mesoporous GSM-CTAB@0.1 material has a superior hydrothermal stability, which can be interesting for further applicationas FCC catalysts.

\subsection{Discussion of GSM and HT}

Based on the results above, the minor amount of water stimulates the crystallization of SAPO-11 molecular sieve [21] and accelerates the formation of surfactant micelles. It is important to note that if CTAB is employed in 
"solvent-free" synthesis, mesopore cannot be formed due to the insufficient amount of water required to form micelles [22]. Besides water is a dispersion medium allowing blending the CTAB micelles with the precursors during the mechanical grinding.

Based on the results reported for samples HT-CTAB@0.1 and GSM-CTAB@0.1 (Table 1), the following advantages of the GSM synthesis approaches are proposed: (1) the organic remains in samples HT-CTAB@0.1 (i.e., 14.3\%) and GSM-CTAB@0.1 (i.e., 12.5\%) are comparable. Considering that a twice amount of DPA was added to regulate the $\mathrm{pH}$ of the precursor mixture used for theHT synthesis, it indicates that excessive DPAwas fully removed during filter. The harmful and expensive organics can be substantially reduced bythe GSM synthesis (the GSM synthesis ofSAPO-11 saved $25 \%$ of DPA). (2) Utilization of inorganic sources in GSM-CTAB@0.1 is $14.2 \%$ higher than HT-CTAB@0.1. (3) Easy synthesis based on grinding of inorganic precursors with organic templates. For example, crystalline precursor is prepared by mixing amorphous feeds with 5 min grinding. (4) Saving water from the synthesis and from the purification of the crystalline products all water required for the synthesises is coming from the raw materials. (5) Increasing crystalline yield of the SAPO-11 product; the yield increases from 63.4 to 77.6wt\% for SAPO-11 synthesize by GSM (i.e., sampleGSM-CTAB@0.1). 
Table 1Startingraw materials, and weight of as synthesized and calcined products.

\begin{tabular}{cccccc}
\hline Samples & $\begin{array}{c}\text { pH of amorphous } \\
\text { mixture }\end{array}$ & $\begin{array}{c}\text { Non-calcined } \\
\text { product/g }\end{array}$ & $\begin{array}{c}\text { Calcined } \\
\text { product/g }\end{array}$ & $\begin{array}{c}\text { Weight loss } \\
\text { during } \\
\text { calcination/\% } \%\end{array}$ & $\begin{array}{c}\text { Utilization of } \\
\text { inorganic } \\
\text { sources/\% } \%\end{array}$ \\
\hline HT-CTAB@0.1 & 5.5 & 5.84 & 5.10 & 14.3 & 63.4 \\
GSM-CTAB@0.1 & $\sim 2.0$ & 2.36 & 2.08 & 12.5 & 77.6 \\
\hline
\end{tabular}

${ }^{\&}$ This value includes DPA used to prepare amorphous materials (i.e., $3.30 \mathrm{~g}$ ) and to regulate pHvalue (i.e., $1.06 \mathrm{~g}$ ) before crystallization.

$\dagger$ The utilization is calculated from the weight ratio of calcinated SAPO- 11 with total inorganicsources (i.e., $\mathrm{Al}_{2} \mathrm{O}_{3}$, $\mathrm{P}_{2} \mathrm{O}_{5}$, and $\left.\mathrm{SiO}_{2}\right)$.

\subsection{Catalytic performance}

$\mathrm{N}$-dodecane is cracked into dry gas (i.e., $\mathrm{C}_{1-2}$ ), liquefied petroleum gas (LPG, i.e., $\mathrm{C}_{3-4}$ ), gasoline fraction (i.e., $\mathrm{C}_{5-11}$ ). Dry gas and LPG are formed from cracking of carbenium ions of $\mathrm{C}_{12}$ and secondary cracking of $\mathrm{C}_{5-11}$ [23]. The conversions of n-dodecane without and with GSM-CTAB@0.1 catalyst are 51.0 and $50.0 \mathrm{wt} \%$, respectively. Based on the acidity of FCC catalysts without and with GSM-CTAB@0.1 in Figure S5, the increasing intensity of Brønsted acid sites (i.e., signal at $\sim 1540 \mathrm{~cm}^{-1}$ ) is attributed to the extra GSM-CTAB@0.1 promoters. Similar conversion indicates that n-dodecane is mainly cracked on strong acid sites, for example with $\mathrm{Y}$ zeolite as a component in FCC catalysts [17].The GSM-CTAB@0.1 has a minor contribution due to a mild acidity, which is supported by a similar coke deposition on catalysts without $(0.85 \mathrm{wt} \%)$ and with $(0.88 \mathrm{wt} \%)$ the GSM-CTAB@0.1 promoter.Adding GSM-CTAB@0.1 decreases the acid strength of the whole catalysts and avoids the secondary cracking of $\mathrm{C}_{5-11}$. 
Yield of $\mathrm{C}_{5-11}$ increases from $\sim 66.0$ to $71.7 \mathrm{wt} \%$ after adding the GSM-CTAB@0.1 as a promoter. A detailed analysis of liquid products suggests that yields of olefin, arene, and cycloparaffin are similar (within the error range) between FCC catalysts without and with GSM-CTAB@0.1 promoter (Figure 8B). Higher yields of n-paraffin and iso-paraffin are attributed to a weak secondary cracking of $\mathrm{C}_{5-11}$ using catalysts with GSM-CTAB@0.1 promoter. The ratio of iso-paraffin/n-paraffin is higher and the yield of iso-paraffin increases from 7.5 to $9.5 \mathrm{wt} \%$. Based on previous research [24], iso-paraffin is generated via two paths: (I) $\beta$-cracking of iso-carbenium ions of $\mathrm{n}$-dodecane and (II) isomerization of intermediates (e.g., n-hexene) on the promoter. Normally, there is a positive correlation between $\beta$-cracking of iso-carbenium ions and the conversion of $\mathrm{n}$-dodecane. Considering the similar conversions of n-dodecane without and with GSM-CTAB@0.1 promoter, contribution of path (I) tothe increase of iso-paraffin is not considered. Therefore, path (II) plays a key role to increase the yield of iso-paraffin. SAPO-11 has a one-dimensional pore channel $(0.39$ $\times 0.64 \mathrm{~nm}$ ). Thekinetic diameter of $\mathrm{n}$-hexene is $0.49 \mathrm{~nm}$ [15], and it can enter the pore of SAPO-11 and converts to its isomer. As for the GSM-CTAB@0.1, introduction of intra-crystalline mesopore increases the exposure of micropore mouth in the bulk of particles, decreases diffusion resistance, and avoids isomers being cracked by the promoter. In order to prove the contribution of the mesopore, GSM-CTAB@0 is prepared and used as a reference promoter. 
GSM-CTAB@0 hassimilar acidity and particle size with

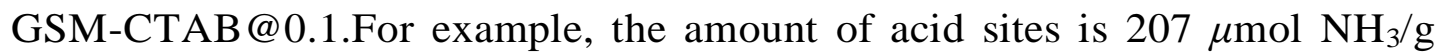
STP for GSM-CTAB @ 0 and $226 \mu \mathrm{mol} \mathrm{NH} \mathrm{NH}_{3} / \mathrm{g} \mathrm{STP}$ for GSM-CTAB @ 0.1. The

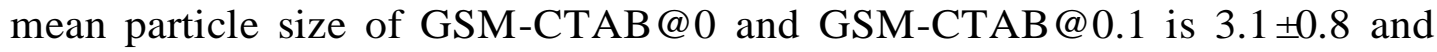
2.2 $\pm 1.4 \mu \mathrm{m}$, respectively (Figure S5). The main difference is the pore structure. GSM-CTAB@0.1 has a higher mesopore volume $\left(0.16 \mathrm{~cm}^{3} / \mathrm{g}\right)$ compared to $\operatorname{GSM}\left(0.09 \mathrm{~cm}^{3} / \mathrm{g}\right)$. Catalytic evaluation results show that no enhancement of isomerization is observed when GSM-CTAB@0 is employed as a promoter (Figure S6). It suggests that absence of mesopores causes a high diffusion resistance of iso-paraffin molecules and a subsequent cracking of iso-paraffin results in a low yield $(6.7 \mathrm{wt} \%)$. The aboveresultsshow that if the GSM-CTAB@0.1 is employed as the RON promoter, it has a great potential to upgrade the quality of gasoline by increasing the yield of iso-paraffins in comparison to pure microporous SAPO-11.
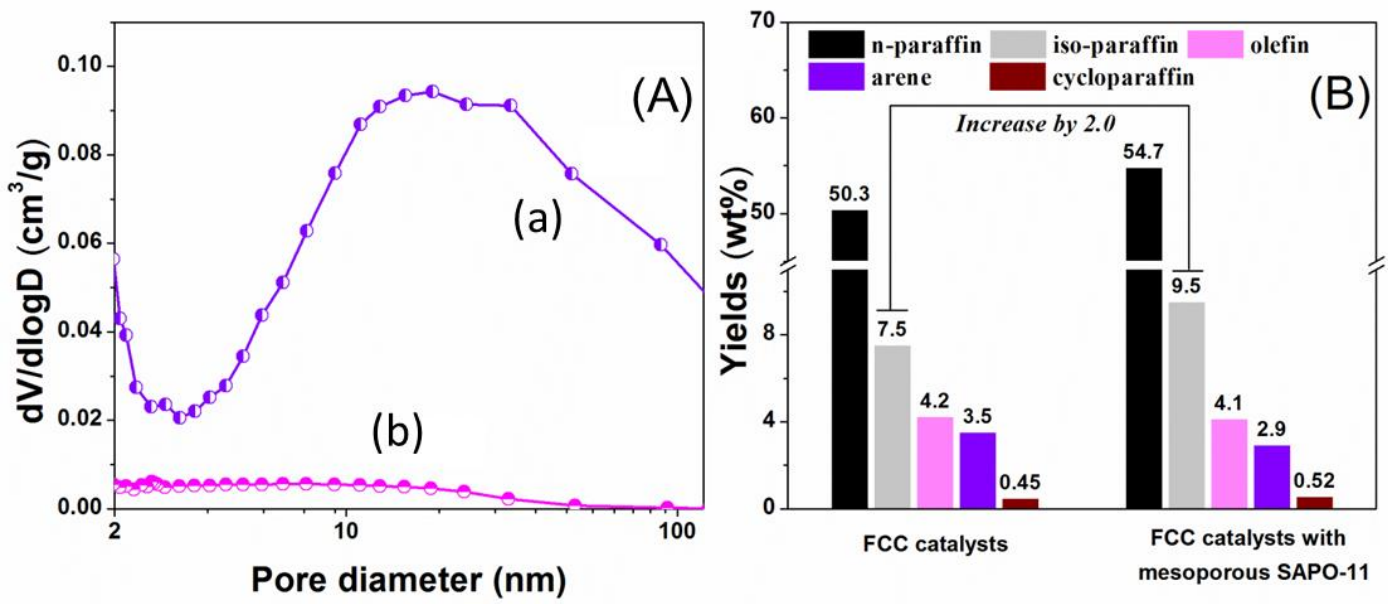

Figure 8. (A):BJH pore size distribution of GSM-CTAB@0.1 (a) and HT-CTAB@0.1 (b) after treatment at $800{ }^{\circ} \mathrm{C}$ for $4 \mathrm{~h}$ under (Relative humidity=100\%). (BJH curves are calculated based on the adsorption isotherms); (B): Yields of n-paraffin, iso-paraffin, olefin, arene, and cycloparaffin in fraction $\mathrm{C}_{5-12}$. (The maximum errors of the 
component concentration for catalysts are n-paraffin $( \pm 0.8 \%)$, iso-paraffin $( \pm 0.5 \%)$, arene $( \pm 0.5 \%)$, olefin $( \pm 0.4 \%)$, and cycloparaffin $( \pm 0.1 \%)$.

\section{Conclusions}

In this work we report on the preparation of hierarchical SAPO-11 molecular sieveby a grinding synthesis method (GSM). The SAPO-11 was used as a FCC isomerization promoter to increasethe iso-paraffinin gasoline.Besides the easy-to-operate and environment friendly characterof the GSM synthesis approach, it is worth noting that the GSM provides an easy route to construct pore structure and decrease the particle size of SAPO-11 molecular sieves simultaneously. The hierarchical pore system contains the inherent micropores, inter-crystalline mesopores, and intra-crystalline mesopores. Intra-crystalline mesoporesare stable at $800{ }^{\circ} \mathrm{C}$ under steam. When added as promoters in fluid catalytic cracking catalysts, the hierarchical SAPO-11 molecular sieve increases the yield of iso-paraffin by enhancing isomerization.Considering the excellent catalytic performance of the hierarchical SAPO-11, the GSM method applied can be considered as a reliable, cost-effective and flexible for the synthesis of hierarchical silicoaluminophosphate molecular sieves in the large-scale production.

\section{Declaration of competing interest}

The authors declare thatnocompeting financialinterests or personal relationships exist to influencethe workin this paper. 


\section{Acknowledgements}

The authors wish to thank Dr Bin Wang in Shaanxi Normal University for the analysis of TEM images. TEMwas carried out in part in the Frederick Seitz MaterialsResearch LaboratoryCentral Research Facilities, University of Illinois. The research was supported byNational Nature Science Foundation of China (Grant No. 21908123, 21978326, and 21991091),China PostdoctoralScience Foundation (2019M662462), Key Technology Research and Development Program of Shandong Province(Grant No.2018GSF117009), Natural Science

Foundation of Shandong Province (ZR2019MB029), Shandong Doctoral Foundation (Grant No. ZR2019BB048),State Key Laboratory of Heavy Oil Processing (SKLOP 201902006). 


\section{References}

[1]E.T.C. Vogt, B.M. Weckhuysen, Fluid catalytic cracking: recent developments on the grand old lady of zeolite catalysis, Chem. Soc. Rev. 44 (2015) 7342-7370.

[2] Q.Q. Yu,H.Y.Sun, H.X. Sun, L. Li, X.C. Zhu, S.Y. Ren, Q.X. Guo, B.J. Shen, Highly mesoporous IM-5 zeolite prepared by alkaline treatment and its catalytic cracking performance, Micropor. Mesopor. Mat. 273 (2019) 297-306.

[3]Y.X. Liu, X. Cui, L. Han, Z.W. Yu, X.M. Liu, Role of fluoride ions in synthesis and isomerization performance of superfine SAPO-11 zeolite, Micropor. Mesopor. Mat. 198 (2014) 230-235.

[4]C.S.L. Narasimhan, J.W. Thybaut, G.B. Marin, P.A. Jacobs, J.A. Martens, J.F. Denayer, G.V. Baron, Kinetic modeling of pore mouth catalysis in the hydroconversion of n-octane on Pt-H-ZSM-22, J. Catal. 220 (2003) 399-413.

[5]Q.M. Sun, N. Wang, R.S. Bai, G.R. Chen, Z.Q. Shi, Y.C. Zou, J.H. Yu, Mesoporogen-free synthesis of hierarchical SAPO-34 with low template consumption and excellent methanol-to-olefin conversion, ChemSusChem. 11 (2018) 3812-3820.

[6]M. Hartmann, A.G. Machoke, W. Schwieger, Catalytic test reactions for the evaluation of hierarchical zeolites, Chem. Soc. Rev. 45 (2016) 3313-3330.

[7]D. Verboekend, T.C. Keller, S. Mitchell, J.P. Ramírez, Hierarchical FAU- and LTA-Type zeolites by post-synthetic design: A new generation of highly efficient base catalysts, Adv. Funct. Mater. 23 (2013) 1923-1934.

[8]Y. Wei, T.E. Parmentier, K.P. de Jong, J. Zečević, Tailoring and visualizing the pore architecture of hierarchical zeolites, Chem. Soc. Rev. 44 (2015) 7234-7261.

[9]K. Möller, T. Bein, Mesoporosity- a new dimension for zeolites, Chem. Soc. Rev. 42 (2013) 3689-3707.

[10]A.G. Machoke, A.M. Beltrán, A. Inayat, B. Winter, T. Weissenberger, N.Kruse, R. Güttel, E. Spiecker, W. Schwieger, Micro/ macroporous system: MFI-type zeolite crystals with embedded macropores, Adv. Mater. 27 (2015) 1066-1070.

[11]H. Li, H.Z. Wu, J.L. Shi, Competition balance between mesoporous self-assembly and crystallization of zeolite: a key to the formation of mesoporous zeolite, J. Alloy. Compd. 556 
(2013) 71-78.

[12]M. Choi, K. Na, J. Kim, Y. Sakamoto, O. Terasaki, R. Ryoo, Stable single-unit-cell nanosheets of zeolite MFI as active and long-lived catalysts, Nature. 461 (2009) 246.

[13]X.X. Wang, G. Li, W.H. Wang, C.Z. Jin, Y.Y. Chen, M, Synthesis, characterization and catalytic performance of hierarchical TS-1 with carbon template from sucrose carbonization, Micropor. Mesopor. Mat. 142 (2011) 494-502.

[14]K.K. Zhu, K. Egeblad, C.H. Christensen, Mesoporous carbon prepared from carbohydrate as hard template for hierarchical zeolites, Eur. J. Inorg. Chem. 2007 (2007) 3955-3960.

[15]Y.X. Liu, Y.C. Lyu, X.X. Zhao, L. Xu, S. Mintova, Z.F. Yan, X.M. Liu, Silicoaluminophosphate-11 (SAPO-11) molecular sieves synthesized via a grinding synthesis method, Chem. Commun. 54 (2018) 10950-10953.

[16] L. Han, Y.X. Liu, F. Subhan, X.M. Liu, Z.F. Yan, Particle effect of SAPO-11 promoter on isomerization reaction in FCC units, Micropor. Mesopor. Mat. 194 (2014) 90-96.

[17]Y.X. Liu, X.M. Liu, L.M. Zhao, Y.C. Lyu, L. Xu, M.J. Rood, L. Wei, Z. Liu, Z.F. Yan, Effect of lanthanum species on the physicochemical properties of La/SAPO-11 molecular sieve, J. Catal. 347 (2017) 170-184.

[18]Z. Chen, C. Fan, L. Pang, S.J. Ming, W. Guo, P. Liu, H.P. Chen, T. Li, One-pot synthesis of high performance $\mathrm{Cu}-\mathrm{SAPO}-18$ catalyst for $\mathrm{NO}$ reduction by $\mathrm{NH}_{3}$-SCR: Influence of silicon content on the catalytic properties of Cu-SAPO-18, Chem. Eng. J. 348 (2018) 608-617.

[19]J.A. Martens, P.J. Grobet, P.A.Jacobs, Catalytic activity and Si, Al, P ordering in microporous silicoaluminophosphates of the SAPO-5, SAPO-11, and SAPO-37 type, J. Catal. 126 (1990) 299-305.

[20]I.A. Bakare, O. Muraza, T. Kurniawan, Z.H. Yamani, E.N. Shafei, A.K. Punetha, K.H. Choi, T. Yokoi, Hydrothermal stability of MTT zeolite in hot water: The role of La and Ce, Micropor. Mesopor. Mat. 233 (2016) 93-101.

[21]M. Matsukata, N. Nishiyama, K. Ueyama, Crystallization of FER and MFI zeolites by a vapor-phase transport method, Micropor. Mesopor. Mat. 7 (1996) 109-117.

[22]L.M. Ren, Q.M. Wu, C.G. Yang, L.F. Zhu, C.J. Li, P.L. Zhang, H.Y. Zhang, X.J. Meng, F.S.Xiao, Solvent-free synthesis of zeolites from solid raw materials, J. Am. Chem. Soc. 134 
(2012) 15173-15176.

[23]A. Ishihara, K. Inui, T. Hashimoto, H. Nasu, Preparation of hierarchical $\beta$ and $\mathrm{Y}$ zeolite-containing mesoporous silica-aluminas and their properties for catalytic cracking of n-dodecane, J. Catal. 295 (2012) 81-90.

[24]Y.X. Liu, Y.C. Lyu, B.J. Wang, Y. Wang, X.M. Liu, M.J. Rood, Z. Liu, Z.F. Yan, Promoter effect of heteroatom substituted AlPO-11 molecular sieves in hydrocarbons cracking reaction, J. Colloid. Interf. Sci. 528 (2018) 330-335. 